\title{
Development of Non-contact Prediction System of Surface Roughness with Medium
}

\author{
Zi Li Xu, a , Long Chen ${ }^{2, b}$ and Song $\mathrm{Lu}^{3, \mathrm{c}}$ \\ ${ }^{1,2,3}$ School of Mechatronic Engineering and Automation, Shanghai University, Shanghai 200072, \\ China; \\ azilixu@shu.edu.cn, b136598843@qq.com, 623884179@qq.com
}

Keywords: roughness measurement, difference compensation, BP neural network, GLCM.

\begin{abstract}
To satisfy the requirement of surface roughness with medium measurement in the machining process, a surface roughness prediction system is developed in this paper. A process classification algorithm and a texture feature difference compensation method are proposed. Four kinds of texture feature including contrast, correlation, energy and homogeneity, are extracted from roughness specimen's clean and containing medium surface by using GLCM (Gray level co-occurrence matrix) texture analysis method. Then the model of BP neural network is built to predict the surface roughness of seven kinds of processing technic. Finally, the experimental results show that the system which is developed based on Matlab GUI can effectively predict roughness of containing medium surface of a workpiece.
\end{abstract}

\section{Introduction}

Non-contact surface roughness measurement is a hot topic in the field of roughness detection. T. Jeyapoovan has researched speckle images of grinding workpiece, and found that their gray mean value, standard deviation and kurtosis are proportional to the values of $\mathrm{Ra}$ [1]. B. Rajamanickam analyzed speckle image in frequency domain to improve the efficiency of image processing using the fast Fourier transform [2]. V.K. Volosyuk applied wavelet transform method to the preprocessing of laser speckle image [3]. Jing Yu claimed that gray level co-occurrence matrix (GLCM) and autocorrelation function could be used to analyze laser speckle images [4]. The majority of above study objects are the surfaces with small roughness value, such as grinding machining surface and so on. However, the measurement outcomes are not precise for large ones. Hence speckle method is not suitable for large values, which leads to certain restrictions for application.

In the process of surface texture extraction, the clean surfaces are chosen in order to remove the interference of medium. S. Palani extracted the clean milling surface features and used fuzzy network to predict roughness values [5]. And Min Li extracted GLCM texture features from clean surface of turning parts and built multiple regression models [6]. M. L. Alves also captured clean surface images of specimen to verify the feasibility of the extraction method of the surface texture of the image [7].

In the cutting machining process, online measuring surface roughness is conducted sometimes. But surface is usually covered with cutting fluid. There may be water vapor in surface when humidity is large. The medium of oil or water vapor will bring a lot of interference for texture feature extraction. Few researchers discussed the surface containing the medium using non-contact measurement.

In this paper, surface texture images are photographed by CCD directly. The texture features are extracted from preprocessed images by using GLCM. The model of BP neural network is built later. A method of texture feature difference compensation is applied to improve the recognition rate of roughness prediction. A machining method classification algorithm is used to classify surfaces of seven kinds of processing technic. Finally a surface with medium roughness prediction system is developed by Matlab. 


\section{GLCM Theoretical Fundamentals}

Gray level co-occurrence matrix proposed by Haralick is a texture analysis method, which reflects the image intensity interval comprehensive information and magnitude of changes in either direction [8].

$p_{\delta}(i, j)$ is the pixel normalized to the joint probability distribution. Four common texture features are shown as follows:

1) Contrast

$$
C O N=\sum_{i} \sum_{j}(i-j)^{2} p_{\delta}(i, j)
$$

Contrast can be described as the clarity of the image. It is the clarity of texture on the visual effects. If the image has more delicate texture, the value of contrast will be larger.

2) Energy

$$
A S M=\sum_{i} \sum_{j}\left[p_{\delta}(i, j)\right]^{2}
$$

Energy is also called angular second moment. It is the gray uniformity of images [9]. The larger the surface roughness is, the greater the energy becomes.

3) Homogeneity

$$
H=\sum_{i} \sum_{j}\left[p_{\delta}(i, j)\right] \log _{2} p(i, j)
$$

Homogeneity is the measure of the randomness of the image content. If the image has no texture, the value of it is almost zero.

4) Correlation

$$
C O R=\left[\sum_{i} \sum_{j}\left(i \cdot j \cdot p_{\delta}(i, j)-\mu_{x} \mu_{y}\right)\right] / \sigma_{x}^{2} \sigma_{y}^{2}
$$

Correlation reflects the degree of similarity in the column and row direction of GLCM elements. In this paper, four features are considered as the texture parameters for extraction.

\section{Texture Features Extraction Process}

Image Acquisition System. The workpiece surface image acquisition system is established in this paper, shown in Figure 1. The system consists of a SX-3 stereoscopic microscope, the Panasonic 5 mega pixel CCD, SENTRY illuminometer, annular LED light source and a PC. The objects of image acquisition are standard roughness specimen which includes seven processing technic i.e. turning, flat milling, vertical milling, planing, external grinding, flat grinding and grinding.

To reduce the random error, the areas of image acquisition are divided into 30 blocks as shown as Figure 2, including 6 rows and 5 columns. The texture features of the resulting values are averaged as the specimen surface texture data.

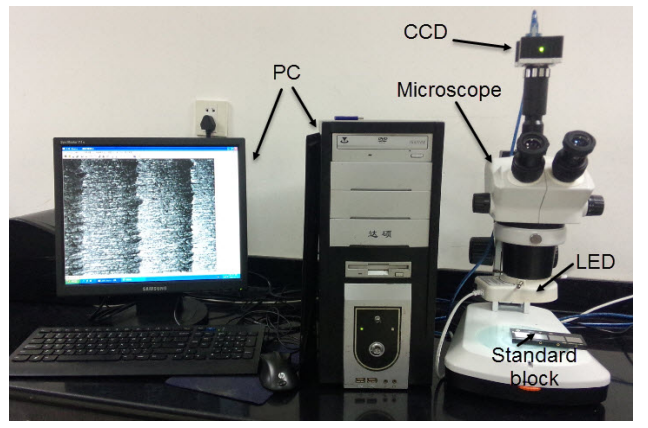

Fig. 1 Surface image acquisition system

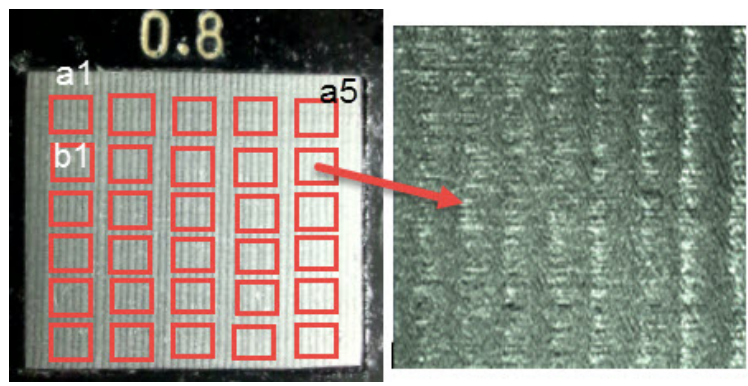

Fig. 2 Flat milling process acquisition areas

The most commonly used parameters of surface height are average roughness Ra. Matlab image processing toolbox is applied in the experiment. Graying, histogram equalization and median filtering pretreatment are used to enhance the texture detail. Features extraction flow diagram is shown in Figure 3. 


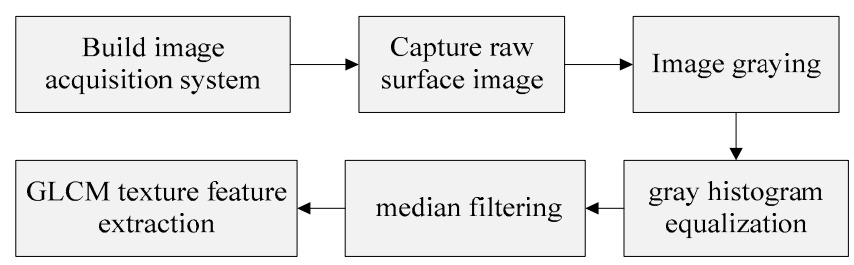

Fig. 3 Features extraction flow diagram

Texture Features Extraction. In order to investigate the influence of oil and water vapor medium on the texture features of workpiece surface, the surfaces are covered with oil medium. The thickness of the oil is about $0.5 \mathrm{~mm}$. In the roughness sample block, the surface of the water vapor is simulated by two kinds of water vapor medium, the diameter of which is about $0.8 \mathrm{~mm}$ and $0.4 \mathrm{~mm}$ respectively. When $\mathrm{Ra}=0.8 \mathrm{~mm}$, texture images of flat milling roughness sample surface with oil and two kinds of water vapor medium are shown in Figure 4.

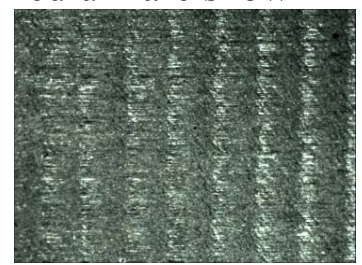

a) Oil medium

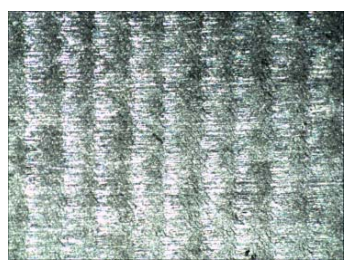

b) Small kind of vapor

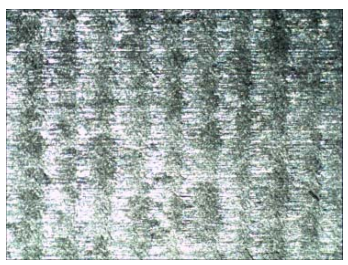

c) Large kind of vapor

Fig. 4 Three kinds of medium images of flat milling process

Four texture feature values are calculated by the optimized parameters: $\mathrm{d}=5, \mathrm{~g}=32$ and $\theta=90^{\circ}$. For example, the feature values of the roughness sample blocks of flat milling process in the clean, oil and water vapor surface are shown in Table 1.

Table 1 Features of different surface states of flat milling process

\begin{tabular}{|c|c|c|c|c|c|c|c|}
\hline $\mathrm{Ra}=6.3 \mu \mathrm{m}$ & $\begin{array}{l}\text { Clean } \\
\text { surface }\end{array}$ & Oil & $\begin{array}{l}\text { Small } \\
\text { vapor }\end{array}$ & $\begin{array}{l}\text { Large } \\
\text { vapor }\end{array}$ & $\begin{array}{c}\text { Oil } \\
\text { compensation }\end{array}$ & $\begin{array}{l}\text { Small vapor } \\
\text { compensation }\end{array}$ & $\begin{array}{l}\text { Large vapor } \\
\text { compensation }\end{array}$ \\
\hline Contrast & 36.931 & 36.063 & 35.032 & 31.525 & 0.868 & 1.899 & 5.406 \\
\hline Correlation & 0.7655 & 0.7746 & 0.7908 & 0.8111 & -0.0091 & -0.0253 & -0.0456 \\
\hline Energy & 0.0020 & 0.0022 & 0.0024 & 0.0030 & -0.0002 & -0.0004 & -0.001 \\
\hline $\begin{array}{c}\text { Homogeneity } \\
\mathrm{Ra}=3.2 \mu \mathrm{m}\end{array}$ & 0.3243 & 0.3308 & 0.3436 & 0.3480 & -0.0065 & -0.0193 & -0.0237 \\
\hline Contrast & 51.929 & 50.064 & 43.801 & 41.190 & 1.865 & 8.128 & 10.739 \\
\hline Correlation & 0.6585 & 0.6761 & 0.7163 & 0.7390 & -0.0176 & -0.0578 & -0.0805 \\
\hline Energy & 0.0016 & 0.0018 & 0.0020 & 0.0023 & -0.0002 & -0.0004 & -0.0007 \\
\hline $\begin{array}{c}\text { Homogeneity } \\
\mathrm{Ra}=1.6 \mu \mathrm{m}\end{array}$ & 0.2828 & 0.2994 & 0.3093 & 0.2944 & -0.0166 & -0.0265 & -0.0116 \\
\hline Contrast & 60.317 & 59.868 & 57.701 & 55.633 & 0.449 & 2.616 & 4.684 \\
\hline Correlation & 0.5928 & 0.6036 & 0.6152 & 0.6305 & -0.0108 & -0.0224 & -0.0377 \\
\hline Energy & $\begin{array}{c}0.0015 \\
2\end{array}$ & $\begin{array}{c}0.0016 \\
5\end{array}$ & 0.00164 & 0.00170 & -0.00013 & -0.00012 & -0.00018 \\
\hline $\begin{array}{c}\text { Homogeneity } \\
\mathrm{Ra}=0.8 \mu \mathrm{m}\end{array}$ & 0.2700 & 0.2745 & 0.2774 & 0.2811 & -0.0045 & -0.0074 & -0.0111 \\
\hline Contrast & 64.655 & 61.711 & 60.808 & 53.765 & 2.944 & 3.847 & 10.89 \\
\hline Correlation & 0.5654 & 0.5884 & 0.5963 & 0.6180 & -0.023 & -0.0309 & -0.0526 \\
\hline Energy & $\begin{array}{c}0.0014 \\
9\end{array}$ & $\begin{array}{c}0.0015 \\
2\end{array}$ & 0.00154 & 0.00158 & -0.00003 & -0.00005 & -0.00009 \\
\hline Homogeneity & 0.2656 & 0.2683 & 0.2711 & 0.2865 & -0.0027 & -0.0055 & -0.0209 \\
\hline
\end{tabular}

From the results of features data analysis, it could be concluded that the contrast is decreased with the addition of roughness, which indicates that the texture of the image is coarser when $\mathrm{Ra}$ is increased.

The homogeneity is increased with the addition of roughness, which indicates that the texture of the image is more random when $\mathrm{Ra}$ is increased.

The energy is increased with the addition of the roughness, which indicates that the gray distribution of the image is more uniform with the increase of Ra value. 
The correlation is increased with the addition of roughness, which indicates that the texture direction of the image is more obvious when $\mathrm{Ra}$ is increased. Table 1 is also indicated that the instability of the texture features of the image is increased by the oil and water vapor medium. Similarly, the same conclusion can be obtained by analyzing the texture features of the other process technic simples.

\section{Process Classification Recognition Algorithm}

In the process of measurement, surveyors need to know the real information of surface machining method. In order to make the prediction system recognize the machining categories automatically, a classification algorithm is proposed based on the distribution of texture features extracted from the surface covered oil, or water vapor or other medium. To minimize the impact of compression on image quality, the calculating parameters are $\mathrm{d}=1, \mathrm{~g}=256$ and $\theta=0^{\circ}$. The features distribution of seven kinds of process technic is shown in Table 2.

Table 2 Features distribution of seven kinds of process technic

\begin{tabular}{ccccc}
\hline \multirow{2}{*}{ Process technic } & \multicolumn{4}{c}{ The texture features distribution of clean, oil or water vapor surface } \\
& Contrast & Correlation & Energy & Homogeneity \\
\hline Turning & $(259.32,632.42$ & $(0.904,0.974$ & $(0.0019,0.0059$ & $(0.552,0.693$ \\
& ) & ) & ) & ) \\
Planing & $(366.39,615.17$ & $(0.891,0.999$ & $(0.0013,0.0029$ & $(0.153,0.337$ \\
& ) & ) & ) & ) \\
Flat milling & $(140.27,329.67$ & $(0.903,0.994$ & $(0.0011,0.0028$ & $(0.235,0.425$ \\
Vertical milling & $(16.76,129.69)$ & $(0.986,0.999$ & $(0.0034,0.0098$ & $(0.474,0.645$ \\
Flat grinding & $(201.55,650.98$ & $(0.911,0.987$ & $(0.0036,0.0090$ & $(0.386,0.556$ \\
External grinding & $(296.25,563.91$ & $(0.901,0.999$ & $(0.0051,0.0069$ & $(0.211,0.355$ \\
Grinding & $(1.16,109.80)$ & $(0.802,0.988$ & $(0.0011,0.0037$ & $(0.098,0.439$ \\
\hline
\end{tabular}

According to the features distribution of clean, oil and water vapor medium surface of each process, a classification algorithm for processing technology can be obtained. Classification algorithm flow chart is shown in Figure 5.

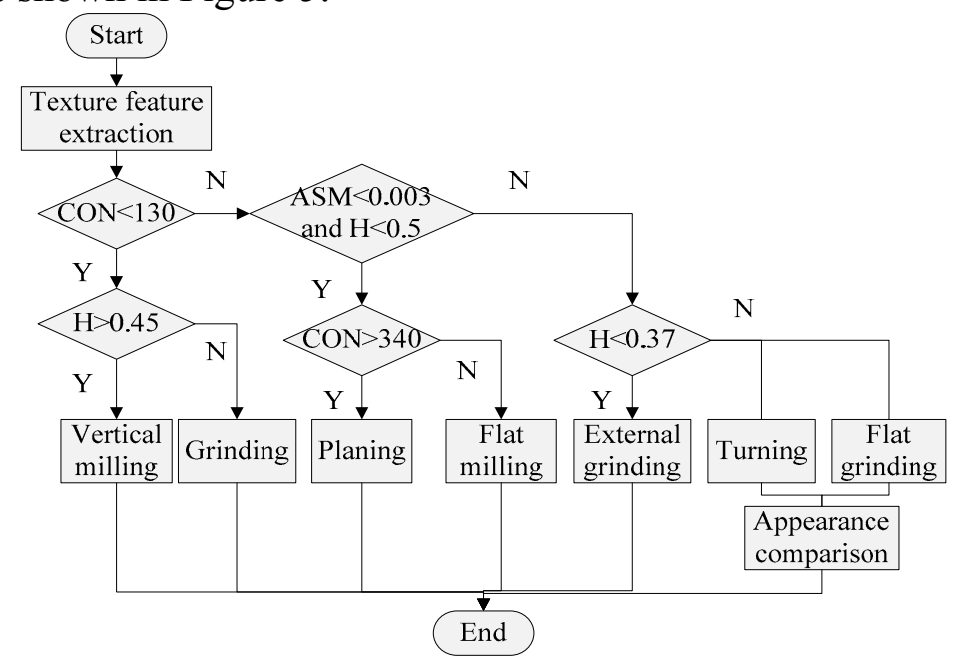

Fig. 5 Classification algorithm flow chart

From Table 2, it can be seen that vertical milling, grinding, planing, flat milling and external grinding process can be separated by the contrast, energy and homogeneity. However, the flat grinding and turning processes with different surface states cannot be well separated by features. Due to the surface of turning process is a circumference surface and flat grinding process surface is a plane 
one, it is easy to judge from the appearance of the workpiece when the surface is from turning or flat grinding process.

\section{BP Neural Network Prediction}

BP neural network is a widely used forecasting model [10]. Four texture features of seven process clean surface are the training samples. BP neural network is established to predict the roughness of seven kinds of machining method. Network target error is 0.01 and the classical gradient descent method is used for the learning method. Neural network training error curve as shown in Figure 6. The neural network in the training to 112th step has reached the goal error of 0.01 . There are 30 prediction samples for the grinding process. There are 40 prediction samples for the other processes respectively. A total of 270 simples of seven kinds of processes are predicted before and after compensation. The results of BP network prediction recognition are shown in Table 3.

Table 3 Recognition rate before and after compensation

\begin{tabular}{ccccccc}
\hline \multirow{2}{*}{ Process } & \multicolumn{2}{c}{ Oil } & \multicolumn{3}{c}{ Small vapor } & \multicolumn{2}{c}{ Large vapor } \\
& Before & After & Before & After & Before & After \\
\hline Turning & $90 \%$ & $93 \%$ & $80 \%$ & $93 \%$ & $70 \%$ & $88 \%$ \\
Planing & $85 \%$ & $90 \%$ & $70 \%$ & $95 \%$ & $70 \%$ & $90 \%$ \\
Flat milling & $83 \%$ & $93 \%$ & $68 \%$ & $88 \%$ & $62 \%$ & $88 \%$ \\
Vertical milling & $80 \%$ & $87 \%$ & $78 \%$ & $88 \%$ & $67 \%$ & $90 \%$ \\
Flat grinding & $75 \%$ & $93 \%$ & $73 \%$ & $90 \%$ & $65 \%$ & $85 \%$ \\
External grinding & $75 \%$ & $93 \%$ & $77 \%$ & $88 \%$ & $55 \%$ & $90 \%$ \\
Grinding & $86 \%$ & $94 \%$ & $80 \%$ & $94 \%$ & $66 \%$ & $87 \%$ \\
\hline
\end{tabular}

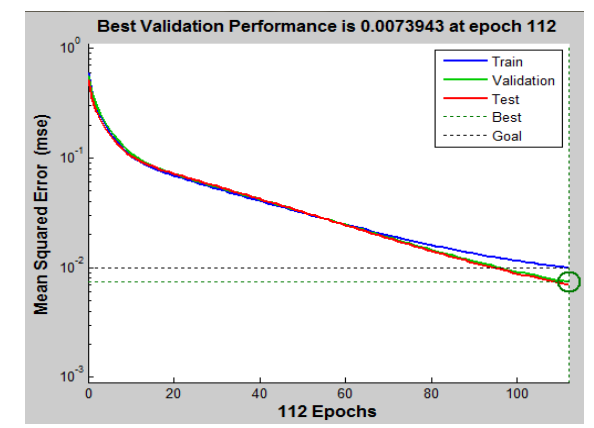

Fig. 6 Network training curve

From Table 3, the recognition rate of prediction roughness is $60-90 \%$ before compensation. However, the surface roughness of the external grinding process has the lowest rate, only $55 \%$.

Due to certain interfere generated by oil and water vapor medium, recognition of roughness is not very precise. In this paper, the following method is presented to reduce the influence of medium.

Firstly, the difference of the texture feature value of the oil or water vapor medium and the one of clean surface is used as the compensation value for the texture features. The compensation values of the surface oil or the vapor medium of the roughness are listed as shown in Table 1.Secondly, the texture of the surface of prediction samples containing oil or water vapor is extracted and calculated by the image acquisition system, and the prediction sample data is obtained. Thirdly, the prediction data and the corresponding compensation value are added to the sample data after compensation. Finally, the optimized BP neural network model is built to predict the surface roughness by the simple data after the compensation.

After using the difference compensation method, the accuracy of the roughness of the workpiece in three different surface states can be improved efficiently as shown in Table 3. 


\section{Surface Roughness Prediction System}

In order to verify the feasibility and validity, the surface roughness prediction system is developed based on the Matlab graphic user interface.

This system mainly consists of three functional modules. The first one is the texture feature extraction module. The second one is BP neural network prediction module. The third one is the result display module. The main interface of the roughness prediction system is shown in Figure 7. System operation procedure flow chart is shown in Figure 8.

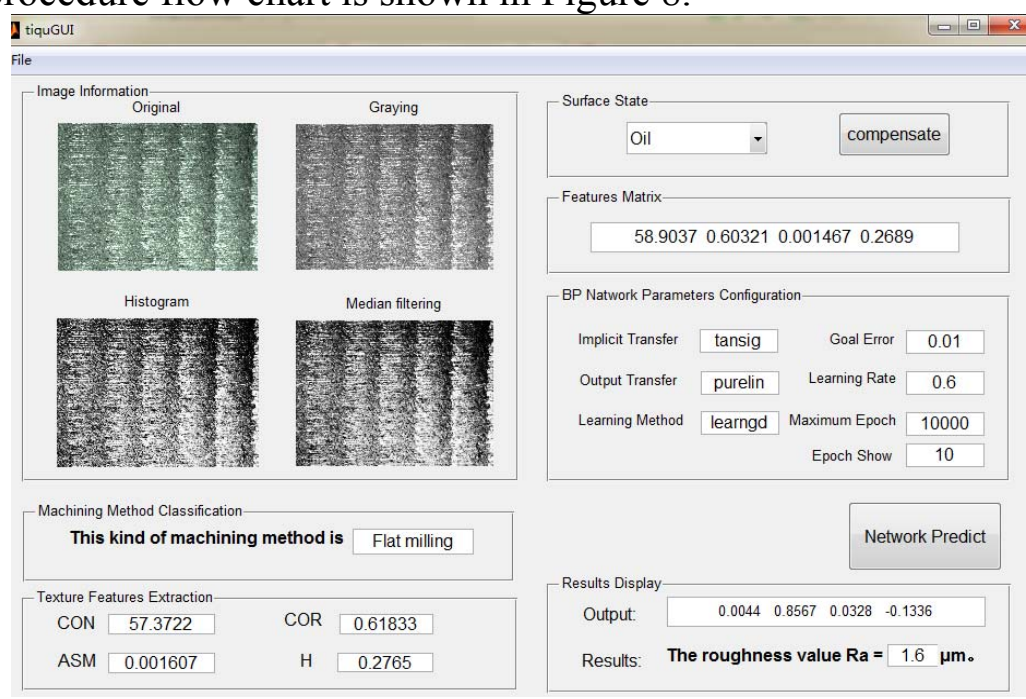

Fig.7 Main interface of the roughness prediction system

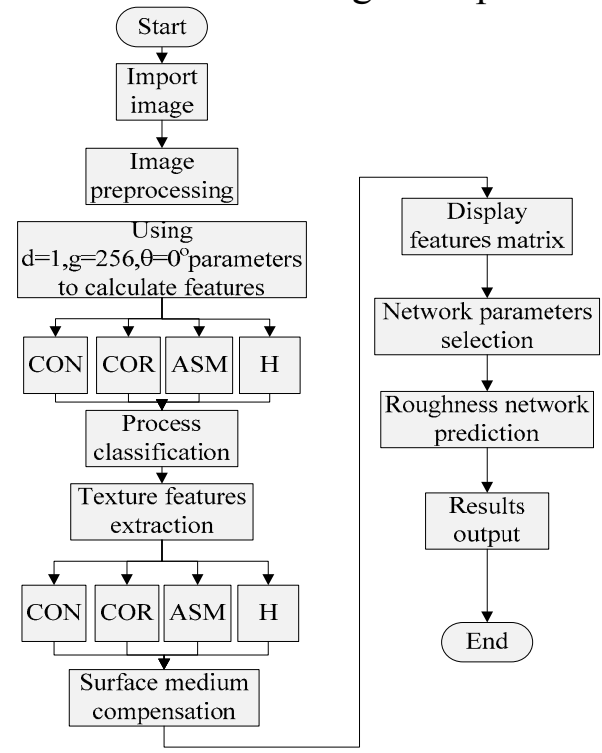

Fig.8 System operation procedure flow chart

For example, firstly, a texture image of flat milling machining method is imported into system to start this prediction. The system can automatically output the process category according to the classification algorithm. Secondly, the type of surface medium should be chosen according to the actual situation of the surface. The texture features of the surface are displayed at the prediction interface. Thirdly, BP network predictive parameters should be selected, such as target error, learning rate and so on. Finally, surface prediction results are shown after pressing the BP network forecast button.

From the result of prediction system, it can be seen that the result of the prediction is $\mathrm{Ra}=1.6 \mathrm{~mm}$, which is consistent with the actual roughness of this image. Meanwhile, the results of other processes are matching well with ideal outcomes according above steps. Limited to space, this paper only lists the results of the plain milling process prediction. 


\section{Summary}

A containing medium surface roughness prediction system is finally developed based on Matlab GUI in this paper. The research results of this paper are as follows:

1) The texture feature difference compensation method proposed in this paper can effectively reduce the interference of water vapor and oil medium to texture image, and improve the accuracy of detection of water vapor and oil medium workpiece surface roughness.

2) A process technic classification algorithm proposed in this paper can effectively classify the containing oil and water vapor medium surface of the seven process technic.

3) The system can be used to predict the surface roughness of seven kinds of process technic with oil and water vapor medium. The application range of features difference compensation method is wider than that of the laser speckle method.

However, there are also many aspects need to be further studied. In the production process of the workpiece, the effect of various medium or residues on the surface of the workpiece is complicated. Only two medium i.e. water vapor and oil are simulated in this paper. The texture difference compensation method of workpiece surface residues in other media, such as dust and emulsion on the influence of rough detection can be further explored and researched.

\section{References}

[1] T.Jeyapoovan, M.Murugan. Statistical Analysis of Surface Roughness Measurements Using Laser Speckle Images[C].World Congress on Information and Communication Technologies, Trivandrum, India, 2012, pp: 378-382.

[2] B.Rajamanickam. Application of Fast Fourier Transform in Laser Speckle Image Pattern Correlation Technique for the Metrological Measurement[C].4 ${ }^{\text {th }}$ International Conference on Advanced Computing, Chennai, India, 2012, pp: 1-4.

[3] V.K.Volosyuk. Application of Wavelet Transform For Processing Speckle Patterns in the Analyzing of Surface Roughness[C]. International Kharkov Symposium on Physics and Engineering of Microwaves, Millimeter and Submillimeter Waves, Kharkiv, Ukraine, 2010, pp: 1-3.

[4] Jing Yu, Chen Bo Zhou, Shu Lin Zhao. Two methods of texture study of surface roughness by laser speckle technology [J].Science and Technology Information. 2012, No.33,pp:56-57.

[5] S. Palani. Prediction of surface roughness in end milling process by machine vision using neuro Fuzzy Network[C]. International Conference on Science Engineering and Management Research, Chennai, India, 2014,pp:1-5.

[6] Min Li. Surface roughness measurement based on image texture analysis[C]. $7^{\text {th }}$ International Congress on Image and Signal Processing, Dalian, China, 2014, pp: 514-519.

[7] M. L. Alves. Evaluation of surface roughness standards applying haralick parameters and artificial neural networks[C]. 19th International Conference on Systems, Signals and Image Processing. Vienna, Austria, 2012, pp:452-455.

[8] M. Haralick. Textural features for image classification[J]. Systems, Man and Cybernetics. 1973, Vol.3, No.6, pp: 610-621.

[9] Shu Liu, Qi Gang Jiang. Research on texture feature description of gray level co-occurrence matrix[J]. Science and technology and Engineering. 2012,Vol.12, No.33, pp:8909-8913.

[10] Yan Sun, Mao Xiang Lang. Optimization for railway freight transport based on BP Neural Network[C]. International Conference on Mechatronic Sciences, Electric Engineering and Computer. Shengyang, China, 2013,pp:2192-2199. 